\title{
Immobilization of Enzyme on Polymer Surface
}

Lei Shen, Kenneth Chun Kuen Cheng, McKenna Schroeder, Pei Yang, Neil Marsh,* Joerg

$$
\text { Lahann, * Zhan Chen* }
$$

${ }^{1}$ Department of Chemistry and ${ }^{2}$ Department of Chemical Engineering, University of Michigan, Ann Arbor, Michigan 48109, United States

*Corresponding Authors: nmarsh@umich.edu, lahann@umich.edu, zhanc@umich.edu 


\begin{abstract}
We successfully immobilized enzymes onto polymer surfaces via covalent bonds between cysteine groups of the enzyme and dibromomaleimide functionalities present at the polymer surface. In this work, we used nitro-reductase (NfsB) as a model enzyme molecule. The polymers were prepared by chemical vapor deposition (CVD) polymerization, resulting in surfaces with dibromomaleimide groups. NfsB variants were engineered so that each NfsB molecule only has one cysteine group on the enzyme surface. Two different NfsB constructs were studied, with cysteines at the positions of $\mathrm{H} 360$ and V424, respectively. A combination of sum frequency generation (SFG) vibrational and attenuated total reflectance Fourier transformed infrared (ATR-FTIR) spectroscopies were used to deduce the orientation of the immobilized enzymes on the surface. It was found that the orientation of the immobilized enzymes is controlled by the position of the cysteine residue in the protein. The NfsB H360C construct exhibited a similar orientational behavior on the polymer surface as compared to that on the selfassembled monolayer surface, but the NsfB V424C construct showed markedly different orientations on the two surfaces.
\end{abstract}




\section{Introduction}

Surface immobilized enzymes have been proven valuable in many important applications including biosensors[1-4], anti-fouling coatings[5, 6] as well as for chemical feedstock production and in fuel cell design[7,8]. When tethered to a surface, both enzyme activity and stability are often significantly altered by their interaction with the surface through mechanisms that are not fully understood.

Enzymes may be either physically (non-covalently) adsorbed or covalently immobilized onto a surface. It has been extensively reported that chemical immobilization leads to more stable enzymes, which may adopt preferred orientations and may have improved useful lifetimes [4, 9-11]. A variety of methods exist to covalently attach proteins to surfaces. In our work we typically utilize cysteine residues engineered at appropriate positions on the enzyme surface that react specifically with maleimide functionalized surfaces. Recently, we found that the dibromomaleimide functional group can be used as an alternative strategy for reversibly binding to cysteine residues[12].

Previously we have demonstrated the ability to control the surface orientation of nitroreductase (NfsB) covalently tethered through cysteine-maleimide linkages on a selfassembled monolayer (SAM) surface[11]. NfsB is a homo-dimeric enzyme with two active sites located at the interface between two monomers [13-16].-In our previous experiments, we used a NfsB construct in which the two subunits were genetically fused to produce a single protein. This allowed us to introduce a single surface cysteine residue in one or other subunit [9]. The NfsB-V424C construct positions the cysteine residue near one active site resulting in a protein orientation that presents one active site towards bulk solution and one active site towards the 
tethering surface (Figure 1). The NfsB-H360C construct places the surface cysteine residue in the middle of one monomer resulting in a protein orientation that presents both active sites equally towards bulk solution and parallel to the tethering surface. The orientation of these surface-immobilized enzymes was experimentally determined by a combination of SFG and ATR-FTIR spectroscopies [9]. This approach has been widely applied by our laboratory and others to study the interactions of biological molecules with a range of surfaces[10] [17-22] [23, 24].

Our previous studies of immobilized enzymes have used SAM surfaces [10, 11, 25] as these provided an ordered, chemically well-defined model surface to study enzyme orientation and activity. However, the application of SAMs surfaces is limited because SAM cannot be formed on many substrates. Previously, we also studied immobilized peptide molecules on spincoated polystyrene-maleimide surfaces[26, 27]. Polymer surfaces are more versatile and can be more practically used in many applications. However, spin-coating is usually used on flat surfaces and it is difficult to use substrates with irregular shapes.

We have previously developed a chemical vapor deposition (CVD) polymerization technique that is capable of depositing a variety of reactive polymer thin film coatings onto a broad range of materials, including complex 3D structures[28-36]. These polymer coatings contain the same backbone polymer chains, poly-p-xylylene (PPX), with different reactive functional moieties substituted on the aromatic parts of the backbone polymer chains. These polymer coatings allow for surface immobilization of biomolecules, such as proteins, peptides, DNAs and carbohydrates[37-42]. In this study, we for the first time determined the orientation of NfsB tethered onto thiol-reactive PPX coatings, poly[4-(3,4-dibromomaleimide)-p-xylylene-co- 
p-xylylene]. We compared the results of immobilized enzymes on the polymer surfaces prepared by CVD to those previously obtained from enzymes immobilized on the SAM surface[10, 11].

\section{Experimental}

\subsection{CVD polymer surface preparation}

The polymer surface presenting dibromomaleimide functionality was prepared by CVD (scheme 1). The thiol-reactive polymer coating, poly[4-(3,4-dibromomaleimide)-p-xylylene-cop-xylylene] 2, was deposited on the $\mathrm{CaF}_{2}$ prisms (for SFG study) or ZnSe crystals (for ATRFTIR study) via CVD polymerization of 4-(3,4-dibromomaleimide)[2.2]paracyclophane 1 . The CVD polymerization was performed using the following procedure: The $\mathrm{CaF}_{2}$ prisms or $\mathrm{ZnSe}$ crystals were fixed in a custom-built CVD chamber at a temperature of $13^{\circ} \mathrm{C} .50 \mathrm{mg}$ of dimer 1 was loaded into the sublimation zone and was sublimed at $\sim 120^{\circ}$. Following pyrolysis (at $750^{\circ} \mathrm{C}$ ), the activated dimer vapor was transferred, by a stream of argon flow, into the deposition chamber, and was deposited and polymerized simultaneously on the $\mathrm{CaF}_{2}$ prisms or $\mathrm{ZnSe}$ crystals. The whole CVD process was operated at 0.1 mbar. More details about the CVD polymer preparation and characterization were reported in a different article[12].

\subsection{SFG and ATR-FTIR experiments}

SFG vibrational spectroscopy has been widely introduced previously in excellent publications[17, 43-50] and will not be repeated here. SFG spectra were acquired using a commercial SFG spectrometer from Ekspla Inc. The detailed description of the picosecond SFG setup has been presented before[26, 51, 52]. SFG spectra were obtained by scanning the input infrared laser wavelength at $5 \mathrm{~cm}^{-1}$ per step and averaging 100 laser shots per data point in the amide I frequency region $\left(1500-1800 \mathrm{~cm}^{-1}\right)$. The SFG spectra were then calibrated by the non- 
resonant signal from the quartz window in the same frequency region to compensate for the frequency dependence of the input infrared and time-dependent visible laser intensities, and fitted by a standard spectral fitting program. Compared to the previous experiments on the immobilized enzymes on SAM surfaces, here we used a lower concentration TCEP in NfsB solution since TCEP may damage the polymer surface by breaking the di-bromal bond. Other than this, we basically followed the same experimental procedure as we adopted when we investigated the immobilized NfsB on SAM.[9]

For the ATR-FTIR experiments, similar protocols to those used in a previous study of immobilized NfsB on a SAM surface [9] were adopted here to study NfsB immobilized on the polymer surface. For the polarized ATR-FTIR experiments, NfsB molecules were immobilized on to the surface of CVD polymer deposited onto $\mathrm{ZnSe}$ ATR crystal instead of $\mathrm{CaF}_{2}$ prism from a $\mathrm{NfsB}$ solution in $\mathrm{D}_{2} \mathrm{O}$ with a low TCEP concentration as described above. ATR-FTIR measurements were performed using a Nicolet 6700 FTIR spectrometer. To deduce the orientation of NfsB immobilized on the CVD polymer, polarized SFG (typically ssp - spolarized SFG signal, s-polarized visible input, p-polarized IR input, and ppp) and ATR-FTIR (p and s) spectra were collected. These two types of measurements are independent and provide more independent data to more accurately deduce the orientation of the immobilized enzymes.

\subsection{Orientation determination of $\mathrm{NfsB}$ immobilized on polymer surfaces}

As published before, we have developed a computer software package to calculate the SFG second order susceptibility component ratio (e.g., $\chi_{\mathrm{ppp}} / \chi_{\mathrm{ssp}}$ as well as the polarized ATR FTIR dichroic ratio $(\mathrm{p} / \mathrm{s})$ as a function of protein orientation (e.g., defined by the tilt and twist angles)[24, 53]. Based on the experimentally measured data and the above calculated orientation 
dependent values, the most possible orientation angle ranges of interfacial proteins can be deduced.

Previously the above discussed computer program only considered the delta distribution (meaning all the molecules adopt the same orientation) for the orientation angles in the calculations. It could work very well for proteins ordered at interfaces, e.g., membrane associated proteins and enzymes immobilized on SAM surfaces.[8,9,33] Polymer surfaces, on the other hand, can be quite complicated and more disordered, leading to the immobilized enzymes on polymer surfaces exhibiting some disordering. Therefore, it may be necessary to include more complicated orientation distributions in data analysis for immobilized proteins on polymer surfaces, which will be discussed in more detail later.

\section{Results and discussion}

\subsection{SFG analysis}

As shown previously in Scheme 1, CVD polymer coating was deposited on $\mathrm{CaF}_{2}$ prism, presenting dibromomaleimide groups on the surface. The dibromomaleimide surface was firstly placed in contact with a PBS buffer solution and time-dependent SFG signal was monitored at 1650 and $3200 \mathrm{~cm}^{-1}$. After the SFG signal reached a plateau, SFG spectra were acquired from the polymer/buffer solution interface. Then a certain volume of NfsB stock solution was added into the PBS buffer solution to reach a final enzyme concentration of $10.0 \mu \mathrm{M}$. SFG signal was again monitored as a function of time at $1650 \mathrm{~cm}^{-1}$. This signal gradually increased, indicating enzyme immobilization occurred. Again after the signal stopped increase, SFG ssp and ppp spectra in the amide I frequency region were collected from immobilized NfsB molecules at the polymer/NfsB buffer solution interface. The protein solution was then replaced by a PBS buffer solution at least 
three times so that the surface with immobilized enzymes was washed for at least three times. We believe that such a washing process could remove the physically adsorbed protein molecules (if any) so that all the enzymes on the surface are chemically immobilized. SFG spectra were collected again after the washing process. The spectra fitting results were obtained after fitting the SFG spectra collected from the immobilized peptide interface after the buffer washes.

The SFG spectra collected from both NfsB constructs (H360C and V424C, Figure 1) immobilized on the CVD polymer surface are dominated by one single peak in the amide I region (Figure 2), which was assigned to the amide I signal contributed by the alpha helical structure. This peak was observed at about $1650 \mathrm{~cm}^{-1}$. From the spectral fitting results (supporting information), it was found that the amide I signal $\chi_{\mathrm{ppp} / \chi \text { ssp }}$ ratio for $\mathrm{NfsB}(\mathrm{H} 360 \mathrm{C})$ is 1.42 and for $\mathrm{NfsB}(\mathrm{V} 424 \mathrm{C})$ is 1.16. Compared to the measured $\chi_{\mathrm{ppp} /} \chi_{\mathrm{ssp}}$ ratio of $\mathrm{NfsB}$ immobilized on a SAM surface investigated previously,[9] the $\chi_{\text {ppp }} \chi_{\text {ssp }}$ ratio of the NfsB H360C construct immobilized on the polymer surface is similar in value while the ratio for NsfB V424C is much smaller. This may indicate that NfsB H360C has a similar orientation on SAM and on CVD polymer, while NfsB V424C has different orientations on the two surfaces.

\subsection{ATR-FTIR analysis}

ATR-FTIR measurements were carried out using the same procedure as those used to study immobilized NfsB on SAM described before.[9] Different from the SFG measurements which used $\mathrm{CaF}_{2}$ prism substrate, in ATR-FTIR experiments the CVD polymer presenting surface dibromomaleimide was deposited on the surface of ZnSe total reflection crystal. The ATR-FTIR crystal (with polymer coating) was placed in contact with $\mathrm{D}_{2} \mathrm{O}\left(\mathrm{D}_{2} \mathrm{O}\right.$ was used to avoid spectral confusion by $\mathrm{H}_{2} \mathrm{O}$ in the IR experiment), and ATR-FTIR spectra were acquired as 
the background signal. $\mathrm{D}_{2} \mathrm{O}$ in contact with the CVD polymer was then replaced by the same amount of NfsB solution $\left(\sim 10 \mu \mathrm{M}\right.$ in $\left.\mathrm{D}_{2} \mathrm{O}\right)$ and after the CVD polymer surface was in contact with the protein solution for at least one hour, the surface (with immobilized enzymes) was washed by buffer for at least three times to wash off the possible physically adsorbed enzyme molecules. Both s and p polarized ATR-FTIR spectra were collected from the immobilized NfsB mutants at the solid/liquid interface (Figure 3).

ATR-FTIR spectra (Figure 3) were fitted using four peaks contributed by four different secondary structures: beta-sheet $\left(\mathrm{B}_{2}\right.$ mode), random coil, alpha helix and turn structures. We will only use the ratio from the alpha helical structure in the orientation determination. The ratios of the alpha-helical contribution in the $\mathrm{p}$ and s spectra for $\mathrm{NfsB}$ V424C and NfsB H360C are measured to be 1.97 and 1.96 respectively. For the $\mathrm{H} 360 \mathrm{C}$ construct, the ATR-FTIR p/s ratio is similar to that measured from the immobilized NfsB H360C on the SAM surface while the p/s ratio for the NfsB V424 mutant is much higher than that observed from the immobilized NfsB V424C on the SAM surface.[9] Similar to the SFG results, ATR-FTIR results show that NfsB H360C immobilized on a polymer surface may adopt a similar orientation to that immobilized on a SAM, but NfsB V424C likely adopts varied orientations on two different substrates.

\subsection{Enzyme orientations at polymer surfaces}

As we discussed above, we have developed a systematic methodology to determine orientation of protein molecules on surfaces by combined SFG and ATR-FTIR measurements. Such an approach was used to investigate NfsB H360C and V424C immobilized on SAM surfaces.[9] We also discussed above that a SAM surface can be quite ordered, but a polymer surface can be more complicated and disordered. We could determine the orientations of the two 
NfsB constructs immobilized on SAM assuming a delta orientation distribution.[9] Here we firstly again used a delta orientation distribution to determine the orientations of the two immobilized NfsB constructs on CVD polymer surfaces. As we presented above, both SFG and ATR-FTIR measured polarized ratios for the H360C construct immobilized on polymer are very similar to those for NfsB H360C immobilized on SAM. Using the orientation analysis method, we found that the possible orientation angle regions can be found under a delta orientation distribution assumption, as shown in Figure 4. Figure 4 shows that the most likely orientation angle regions (combinations of the tilt and twist angles) are those regions which have small or large tilt angles. These regions are very similar to those deduced for NfsB H360C immobilized on SAM. Figure 5 shows a schematic of NfsB with the most likely orientation on the CVD polymer surface. It is the same as what we expected because the cysteine group is near the surface, reacted with the dibromomaleimide group on the polymer surface. Our combined SFG and ATR-FTIR study here indicated that NfsB H360C exhibits a similar orientation after immobilization to a SAM or the CVD polymer surface.

Using the same approach, we hoped to determine the orientation of another NfsB construct, V424C, immobilized on the CVD polymer surface. As we discussed above, both SFG $\chi_{\text {ppp }} / \chi_{\text {ssp }}$ ratio and ATR-FTIR p/s ratio are different for NfsB V424C immobilized on CVD polymer compared to those of the enzyme immobilized on SAM, perhaps showing that NfsB V424C adopts varied orientations on SAM and polymer surfaces. We tried to determine the most likely orientation angles of NfsB V424C immobilized on the CVD polymer surface using SFG measurements first. However, the measured $\chi_{\text {ppp }} / \chi_{\text {ssp }}$ ratio is lower than the smallest possible value calculated for NfsB V424C assuming a delta orientation distribution. We therefore believe that here the delta orientation distribution assumption is not valid anymore. 
As we discussed above, a polymer surface can be more disordered compared to a SAM surface, therefore the immobilized enzyme molecules on a polymer surface may not adopt the same orientation. We then replaced the delta distribution with a Gaussian distribution function to calculate the measured $\chi_{\mathrm{ppp} /} \chi_{\mathrm{ssp}}$ ratio as a function of $\mathrm{NfsB}$ V424C's average orientation and different orientation distributions. Unfortunately, regardless of the Gaussian orientation distribution width used in the calculation, we were not able to find a possible average orientation angle region since the experimentally measured SFG $\chi_{\mathrm{ppp} /} \chi_{\mathrm{ssp}}$ ratio is still far below the lowest value produced in the calculation using a Gaussian orientation distribution.

We then modified our orientation analysis computer software to replace the Gaussian distribution with a double delta distribution - or two possible orientation angles that the enzyme molecules can adopt after surface immobilization. Since the surface immobilization chemistry is similar for the immobilization of $\mathrm{NfsB}$ to a SAM surface (maleimide-cysteine coupling) and a CVD polymer surface (dibromomaleimide-cysteine coupling), we believe that some of the immobilized NfsB V424C on the CVD polymer surface should adopt a similar orientation compared to that immobilized on the SAM surface. Here, we assume that some of the immobilized enzyme molecules adopt the same orientation on the CVD polymer surface as that on the SAM (tilt angle $=25^{\circ}$, twist angle $=30^{\circ}$ ), while the rest of the enzyme molecules adopt a different orientation on the polymer surface. That is to say, immobilized enzyme molecules adopt a dual delta-orientation distribution. We calculated the SFG $\chi_{\text {ppp } /} \chi_{\text {ssp }}$ ratio as a function of the second orientation tilt and twist angles and with different ratios of the two different orientations. With the measured SFG and ATR-FTIR data, we can determine the possible orientation angle regions of the second orientation, as shown in Figure 6. It is interesting to see that for most of the ratios of the two different orientations, no orientation angle regions can be 
found to satisfy SFG and ATR-FTIR measured data. However, for $40 \%$ or $60 \%$ enzymes adopting an orientation of tilt angle $=25^{\circ}$, twist angle $=30^{\circ}$, possible orientation angle regions for the second orientation could be found, as shown in Figure 6. However, if we carefully examine the possible second orientation, it was found that the second orientation has a tilt angle at $170^{\circ}$ or $\sim 130^{\circ}$. Figure 7 shows the schematics of the enzyme adopting these two orientations Unfortunately the cysteine is not near the surface, so the deduced orientations may not be correct. Perhaps many more complicated multiple orientation distributions (e.g., dual-delta-distribution with different orientation angles, three- or more-delta-distributions) can satisfy the measured SFG and ATR-FTIR data, which is beyond the scope of this research and will not be further studied here.

It is interesting to observe that two NfsB constructs exhibit different orientation behaviors on CVD polymer surfaces. If we represent the $\mathrm{NfsB}$ molecule as a rectangle, the schematics of NfsB H360C and V424C can be displayed in Figure 8. It looks like that the immobilized NfsB H360C has a more stable structure than that of NfsB V424C. This might be the reason which causes a similar structure of immobilized NfsB H360C on SAM and on CVD polymer, while for NfsB V424C, the orientations are different after immobilized to a SAM surface compared to that after immobilized to a CVD polymer.

\section{Conclusions}

For the first time, we applied a combined SFG and ATR-FTIR approach to examine the orientation of proteins immobilized on the surfaces of polymer coatings. It was found that the two NfsB mutants adopted different orientations, when immobilized on CVD based polymer surfaces. NsfB H360C exhibited a similar orientation on the CVD polymer surface compared to 
that on a SAM surface, as characterized by the delta orientation distribution. In contrast, for the NfsB V424C variant, no orientational angle regions could be identified with a delta angle distribution that matched the measured SFG and ATR-FTIR data. We believe that the immobilized NfsB V424C molecules adopted multiple orientations. Under the assumption of a two-delta orientation distribution, two possible distinct orientation angles were deduced. Therefore, on the polymer surfaces, the two NfsB variants could adopt different orientation patterns: one NfsB construct has a delta orientation distribution, while the other mutant adopts a complex multiple-orientation distribution.

Combining this study with our previous results on enzymes immobilized on SAM surfaces, we believe that for the same enzyme with different immobilization sites, they can certainly adopt distinct orientations on the surface. On the SAM surface, they exhibit the delta orientation distribution, due to the uniform structure of the SAM surface, but have different orientation angles.

In this study we showed that NfsB H360C immobilized on a CVD polymer surface adopts a similar orientation to that on a SAM surface. As we discussed above, CVD polymers have more practical applications because they can be coated on any substrate surfaces with different shapes. We demonstrated that it is feasible to control the orientation of an immobilized enzyme (e.g., NfsB H360C) on CVD polymer surfaces by engineering the enzyme with the immobilization site at a proper position.

\section{Acknowledgement}


This research is supported by the Defense Threat Reduction Agency (HDTRA1-11-10019) and the Army Research Office (W911NF-11-1-0251). We thank Dr. Dr. Andrew

Boughton for his help on data analysis.

\section{Supporting Information}

Fitting parameters.

\section{Reference}

[1] T. Wink, S.J. vanZuilen, A. Bult, W.P. vanBennekom, Self-assembled monolayers for biosensors, Analyst, 122 (1997) R43-R50.

[2] N.K. Chaki, K. Vijayamohanan, Self-assembled monolayers as a tunable platform for biosensor applications, Biosensors \& Bioelectronics, 17 (2002) 1-12.

[3] M. Zhou, S. Dong, Bioelectrochemical Interface Engineering: Toward the Fabrication of Electrochemical Biosensors, Biofuel Cells, and Self-Powered Logic Biosensors, Accounts of Chemical Research, 44 (2011) 1232-1243.

[4] J.N. Talbert, J.M. Goddard, Enzymes on material surfaces, Colloid Surf. B-Biointerfaces, 93 (2012) 8-19.

[5] S.M. Olsen, L.T. Pedersen, M.H. Laursen, S. Kiil, K. Dam-Johansen, Enzyme-based antifouling coatings: a review, Biofouling, 23 (2007) 369-383.

[6] J.B. Kristensen, R.L. Meyer, B.S. Laursen, S. Shipovskov, F. Besenbacher, C.H. Poulsen, Antifouling enzymes and the biochemistry of marine settlement, Biotechnology Advances, 26 (2008) 471-481.

[7] M.B. Tu, X. Zhang, A. Kurabi, N. Gilkes, W. Mabee, J. Saddler, Immobilization of betaglucosidase on Eupergit C for lignocellulose hydrolysis, Biotechnology Letters, 28 (2006) 151156.

[8] X.Y. Yang, G. Tian, N. Jiang, B.L. Su, Immobilization technology: a sustainable solution for biofuel cell design, Energy \& Environmental Science, 5 (2012) 5540-5563.

[9] R.C. Rodrigues, A. Berenguer-Murcia, R. Fernandez-Lafuente, Coupling Chemical Modification and Immobilization to Improve the Catalytic Performance of Enzymes, Advanced Synthesis \& Catalysis, 353 (2011) 2216-2238.

[10] Y. Liu, T.L. Ogorzalek, P. Yang, M.M. Schroeder, E.N.G. Marsh, Z. Chen, Molecular Orientation of Enzymes Attached to Surfaces through Defined Chemical Linkages at the SolidLiquid Interface, J Am Chem Soc, 135 (2013) 12660-12669.

[11] L. Shen, M. Schroeder, T.L. Ogorzalek, P. Yang, F.G. Wu, E.N.G. Marsh, Z. Chen, Surface Orientation Control of Site-Specifically Immobilized Nitro-reductase (NfsB), Langmuir, 30 (2014) 5930-5938 . 
[12] A. Ross, H. Durmaz, K. Cheng, X.P. Deng, Y.W. Liu, J. Oh, Z. Chen, J. Lahann, Selective and Reversible Binding of Thiol-Functionalized Biomolecules on Polymers Prepared via Chemical Vapor Deposition Polymerization, Langmuir, 31 (2015) 5123-5129.

[13] G.N. Parkinson, J.V. Skelly, S. Neidle, Crystal structure of FMN-dependent nitroreductase from Escherichia coli B: A prodrug-activating enzyme, Journal of Medicinal Chemistry, 43 (2000) 3624-3631.

[14] A.L. Lovering, E.I. Hyde, P.F. Searle, A.W. Scott, The structure of Escherichia coli nitroreductase complexed with nicotinic acid: Three crystal forms at 1.7 (A)over-circle, 1.8 (A)over-circle and 2.4 (A)over-circle resolution, Journal of Molecular Biology, 309 (2001) 203213.

[15] G.J. Atwell, S. Yang, F.B. Pruijn, S.M. Pullen, A. Hogg, A.V. Patterson, W.R. Wilson, W.A. Denny, Synthesis and structure-activity relationships for 2,4-dinitrobenzamide-5-mustards as prodrugs for the Escherichia coli nfsB nitroreductase in gene therapy, Journal of Medicinal Chemistry, 50 (2007) 1197-1212.

[16] M. Roldan, E. Perez-Reinado, F. Castillo, C. Moreno-Vivian, Reduction of polynitroaromatic compounds: the bacterial nitroreductases, Fems Microbiology Reviews, 32 (2008) 474-500.

[17] T. Weidner, J.S. Apte, L.J. Gamble, D.G. Castner, Probing the Orientation and Conformation of alpha-Helix and beta-Strand Model Peptides on Self-Assembled Monolayers Using Sum Frequency Generation and NEXAFS Spectroscopy, Langmuir, 26 (2010) 3433-3440. [18] J.E. Baio, T. Weidner, L. Baugh, L.J. Gamble, P.S. Stayton, D.G. Castner, Probing the Orientation of Electrostatically Immobilized Protein G B1 by Time-of-Flight Secondary Ion Spectrometry, Sum Frequency Generation, and Near-Edge X-ray Adsorption Fine Structure Spectroscopy, Langmuir, 28 (2012) 2107-2112.

[19] J.E. Baio, T. Weidner, D. Ramey, L. Pruzinsky, D.G. Castner, Probing the orientation of electrostatically immobilized cytochrome $\mathrm{C}$ by time of flight secondary ion mass spectrometry and sum frequency generation spectroscopy, Biointerphases, 8 (2013) 18.

[20] L. Fu, G. Ma, E.C.Y. Yan, In Situ Misfolding of Human Islet Amyloid Polypeptide at Interfaces Probed by Vibrational Sum Frequency Generation, J Am Chem Soc, 132 (2010) 54055412.

[21] L. Fu, Z. Wang, E.C.Y. Yan, Chiral Vibrational Structures of Proteins at Interfaces Probed by Sum Frequency Generation Spectroscopy, International Journal of Molecular Sciences, 12 (2011) 9404-9425.

[22] L. Fu, J. Liu, E.C.Y. Yan, Chiral Sum Frequency Generation Spectroscopy for Characterizing Protein Secondary Structures at Interfaces, J Am Chem Soc, 133 (2011) 80948097.

[23] A.P. Boughton, K. Nguyen, I. Andricioaei, Z. Chen, Interfacial Orientation and Secondary Structure Change in Tachyplesin I: Molecular Dynamics and Sum Frequency Generation Spectroscopy Studies, Langmuir, 27 (2011) 14343-14351.

[24] P. Yang, A.P. Boughton, K.T. Homan, J.J.G. Tesmer, Z. Chen, Membrane Orientation of Gai $\beta 1 \gamma 2$ and G $\beta 1 \gamma 2$ Determined via Combined Vibrational Spectroscopic Studies, J Am Chem Soc, 135 (2013) 5044-5051.

[25] X. Han, Y. Liu, F.-G. Wu, J. Jansensky, T. Kim, Z. Wang, C.L. Brooks, III, J. Wu, C. Xi, C.M. Mello, Z. Chen, Different Interfacial Behaviors of Peptides Chemically Immobilized on Surfaces with Different Linker Lengths and via Different Termini, J Phys Chem B, 118 (2014) 2904-2912. 
[26] X.F. Han, L. Soblosky, M. Slutsky, C.M. Mello, Z. Chen, Solvent Effect and TimeDependent Behavior of C-Terminus-Cysteine-Modified Cecropin P1 Chemically Immobilized on a Polymer Surface, Langmuir, 27 (2011) 7042-7051.

[27] X. Han, J.R. Uzarski, C.M. Mello, Z. Chen, Different Interfacial Behaviors of N- and CTerminus Cysteine-Modified Cecropin P1 Chemically Immobilized onto Polymer Surface, Langmuir, 29 (2013) 11705-11712.

[28] H.Y. Chen, Y. Elkasabi, J. Lahann, Surface modification of confined microgeometries via vapor-deposited polymer coatings, Journal of the American Chemical Society, 128 (2006) 374380.

[29] H.Y. Chen, M. Hirtz, X.P. Deng, T. Laue, H. Fuchs, J. Lahann, Substrate-Independent DipPen Nanolithography Based on Reactive Coatings, Journal of the American Chemical Society, 132 (2010) 18023-18025.

[30] H.Y. Chen, J. Lahann, Surface patterning strategies for microfluidic applications based on functionalized poly-p-xylylenes, Bioanalysis, 2 (2010) 1717-1728.

[31] H.Y. Chen, A.A. McClelland, Z. Chen, J. Lahann, Solventless adhesive bonding using reactive polymer coatings, Anal Chem, 80 (2008) 4119-4124.

[32] H.Y. Chen, J.M. Rouillard, E. Gulari, J. Lahann, Colloids with high-definition surface structures, P Natl Acad Sci USA, 104 (2007) 11173-11178.

[33] X.P. Deng, J. Lahann, Orthogonal Surface Functionalization Through Bioactive VaporBased Polymer Coatings, J Appl Polym Sci, 131 (2014) 40315.

[34] T.D.Y. Kozai, N.B. Langhals, P.R. Patel, X.P. Deng, H.N. Zhang, K.L. Smith, J. Lahann, N.A. Kotov, D.R. Kipke, Ultrasmall implantable composite microelectrodes with bioactive surfaces for chronic neural interfaces, Nat Mater, 11 (2012) 1065-1073.

[35] J.P. Seymour, Y.M. Elkasabi, H.Y. Chen, J. Lahann, D.R. Kipke, The insulation performance of reactive parylene films in implantable electronic devices, Biomaterials, 30 (2009) 6158-6167.

[36] S. Tawfick, X.P. Deng, A.J. Hart, J. Lahann, Nanocomposite microstructures with tunable mechanical and chemical properties, Phys Chem Chem Phys, 12 (2010) 4446-4451.

[37] F. Bally, K. Cheng, H. Nandivada, X.P. Deng, A.M. Ross, A. Panades, J. Lahann, Coimmobilization of Biomolecules on Ultrathin Reactive Chemical Vapor Deposition Coatings Using Multiple Click Chemistry Strategies, Acs Appl Mater Inter, 5 (2013) 9262-9268.

[38] X.P. Deng, T.W. Eyster, Y. Elkasabi, J. Lahann, Bio-Orthogonal Polymer Coatings for CoPresentation of Biomolecules, Macromol Rapid Comm, 33 (2012) 640-645.

[39] X.P. Deng, C. Friedmann, J. Lahann, Bio-orthogonal co-immobilization strategies based on multifunctional chemical vapor deposition polymer coatings, Abstr Pap Am Chem S, 243 (2012). [40] S. Thevenet, H.Y. Chen, J. Lahann, F. Stellacci, A generic approach towards nanostructured surfaces based on supramolecular nanostamping on reactive polymer coatings, Adv Mater, 19 (2007) 4333-4337.

[41] Y.M. Elkasabi, J. Lahann, P.H. Krebsbach, Cellular transduction gradients via vapordeposited polymer coatings, Biomaterials, 32 (2011) 1809-1815.

[42] Y. Zhang, X.P. Deng, E.L. Scheller, T.G. Kwon, J. Lahann, R.T. Franceschi, P.H. Krebsbach, The effects of Runx2 immobilization on poly (epsilon-caprolactone) on osteoblast differentiation of bone marrow stromal cells in vitro, Biomaterials, 31 (2010) 3231-3236.

[43] Y.R. Shen, Surface Properties Probed by $2^{\text {nd }}$-harmonic and sum-frequency generation, Nature, 337 (1989) 519-525. 
[44] X. Zhuang, P.B. Miranda, D. Kim, Y.R. Shen, Mapping molecular orientation and conformation at interfaces by surface nonlinear optics, Physical Review B, 59 (1999) 1263212640.

[45] D.H. Gracias, Z. Chen, Y.R. Shen, G.A. Somorjai, Molecular characterization of polymer and polymer blend surfaces. Combined sum frequency generation surface vibrational spectroscopy and scanning force microscopy studies, Accounts of Chemical Research, 32 (1999) 930-940.

[46] Z. Chen, Y.R. Shen, G.A. Somorjai, Studies of polymer surfaces by sum frequency generation vibrational spectroscopy, Annu Rev Phys Chem, 53 (2002) 437-465.

[47] K.B. Eisenthal, Liquid interfaces probed by second-harmonic and sum-frequency spectroscopy, Chemical Reviews, 96 (1996) 1343-1360.

[48] G.L. Richmond, Molecular bonding and interactions at aqueous surfaces as probed by vibrational sum frequency spectroscopy, Chemical Reviews, 102 (2002) 2693-2724.

[49] L. Baugh, T. Weidner, J.E. Baio, P.-C.T. Nguyen, L.J. Gamble, P.S. Slayton, D.G. Castner, Probing the Orientation of Surface-Immobilized Protein G B1 Using ToF-SIMS, Sum Frequency Generation, and NEXAFS Spectroscopy, Langmuir, 26 (2010) 16434-16441.

[50] Q. Li, C.W. Kuo, Z. Yang, P. Chen, K.C. Chou, Surface-enhanced IR-visible sum frequency generation vibrational spectroscopy, Phys Chem Chem Phys, 11 (2009) 3436-3442.

[51] K.T. Nguyen, S.V. Le Clair, S.J. Ye, Z. Chen, Molecular Interactions between Magainin 2 and Model Membranes in Situ, J Phys Chem B, 113 (2009) 12358-12363.

[52] S. Ye, K.T. Nguyen, Z. Chen, Interactions of Alamethicin with Model Cell Membranes Investigated Using Sum Frequency Generation Vibrational Spectroscopy in Real Time in Situ, J Phys Chem B, 114 (2010) 3334-3340.

[53] A.P. Boughton, P. Yang, V.M. Tesmer, B. Ding, J.J.G. Tesmer, Z. Chen, Heterotrimeric G protein beta(1)gamma(2) subunits change orientation upon complex formation with $\mathrm{G}$ proteincoupled receptor kinase 2 (GRK2) on a model membrane, P Natl Acad Sci USA, 108 (2011) E667-E673. 


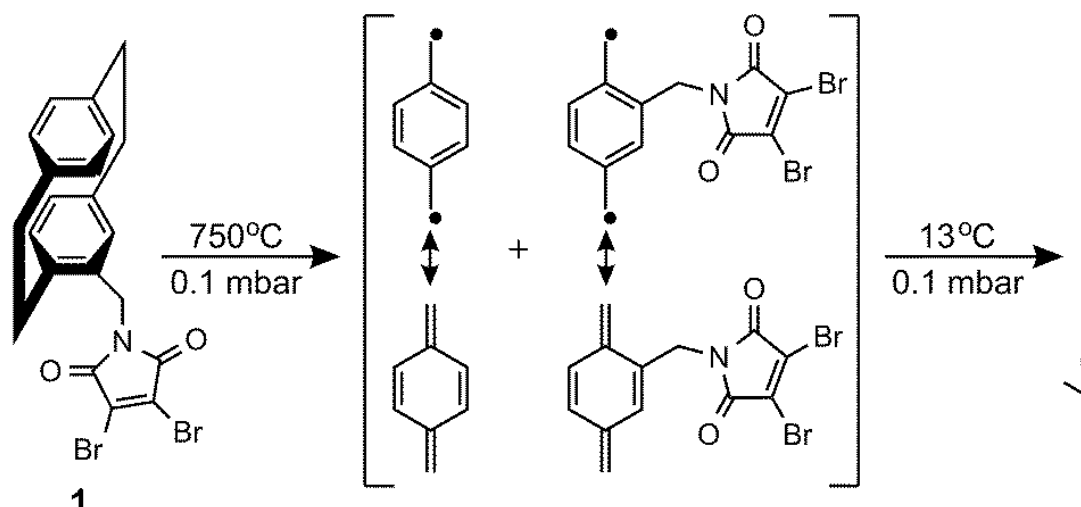<smiles>CCc1ccc(CCC(CC(C)C)c2ccc(CC(C)C)c(CN3C(=O)C(Br)=C(Br)C3=O)c2)cc1</smiles>

2

Scheme 1 The thiol-reactive polymer coating, poly[4-(3,4-dibromomaleimide)-pxylylene-co-p-xylylene] (compound 2), was deposited on a surface via CVD polymerization of 4-(3,4-dibromomaleimide)[2.2]paracyclophane (compound 1).

\section{Active Site}
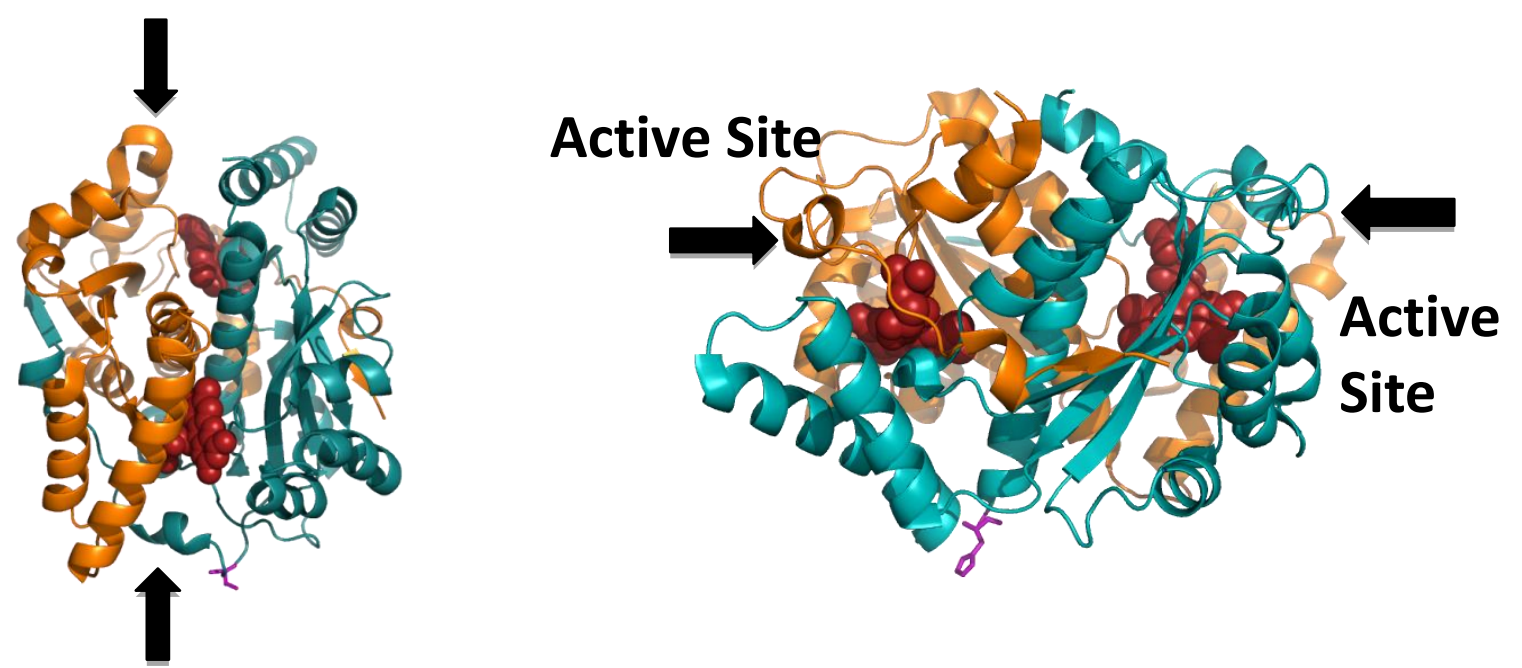

\section{Active Site}

Figure 1 Crystal structures of NfsB V424C (left) and NfsB H360C (right). NfsB holds a dimer structure as depicted. Here in NfsB V424C, the amino acid Valine 424 was replaced by a cysteine residue, while in NfsB H360C, the amino acid Histidine 360 was replaced by cysteine. The thiol group in the cysteine residue can bind to the dibromomaleimide group on CVD polymer surface. Reprinted with permission from Ref. 11. Copyright 2014 American Chemical Society. 

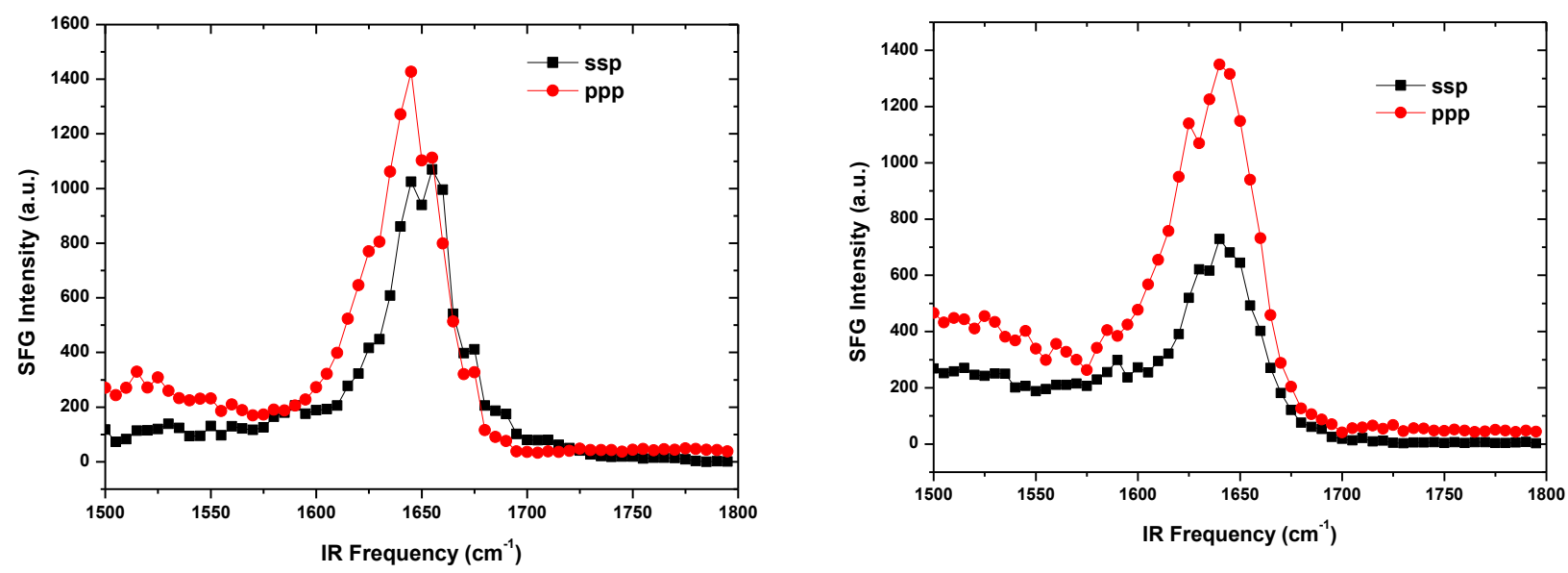

Figure 2 (Left) SFG spectra of NfsB (V424C) immobilized on the CVD polymer surface with different polarization combinations (ssp and ppp); (Right) SFG spectra of NfsB (H360C) immobilized on the CVD polymer surface with different polarization combinations (ssp and ppp).
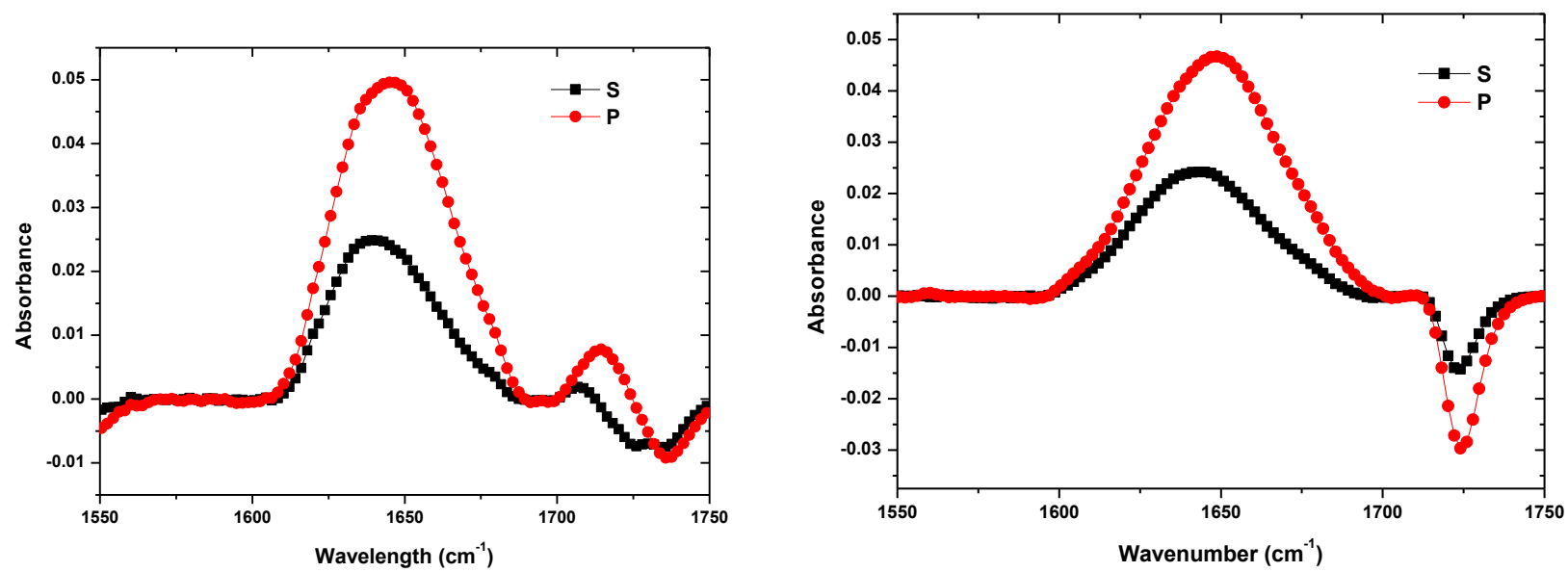

Figure 3 ATR-FTIR s-polarized and p-polarized spectra of NfsB (V424C) (left) and $\mathrm{NfsB}$ (H360C) (right) immobilized on CVD polymer surface. 


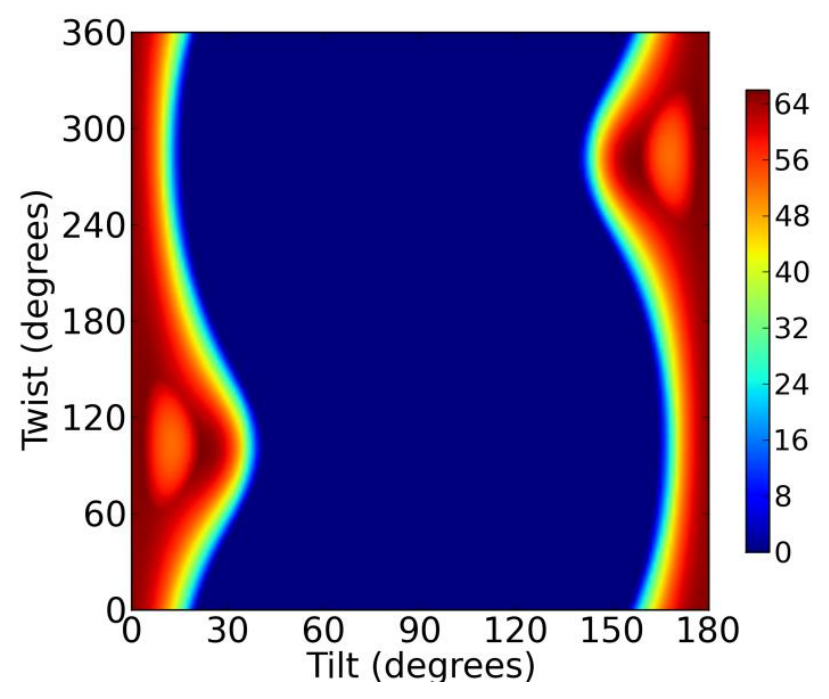

Figure 4 Possible orientations of NfsB H360C immobilized on the CVD polymer surface deduced by combined SFG and ATR-FTIR measurements.

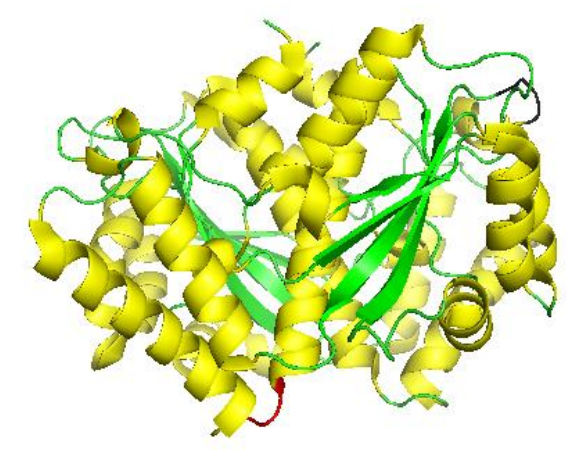

Figure 5 Schematic illustration of final orientation of NsfB (H360C) immobilized on the polymer surface $(150,280)$. Cysteine group is labeled in red. 

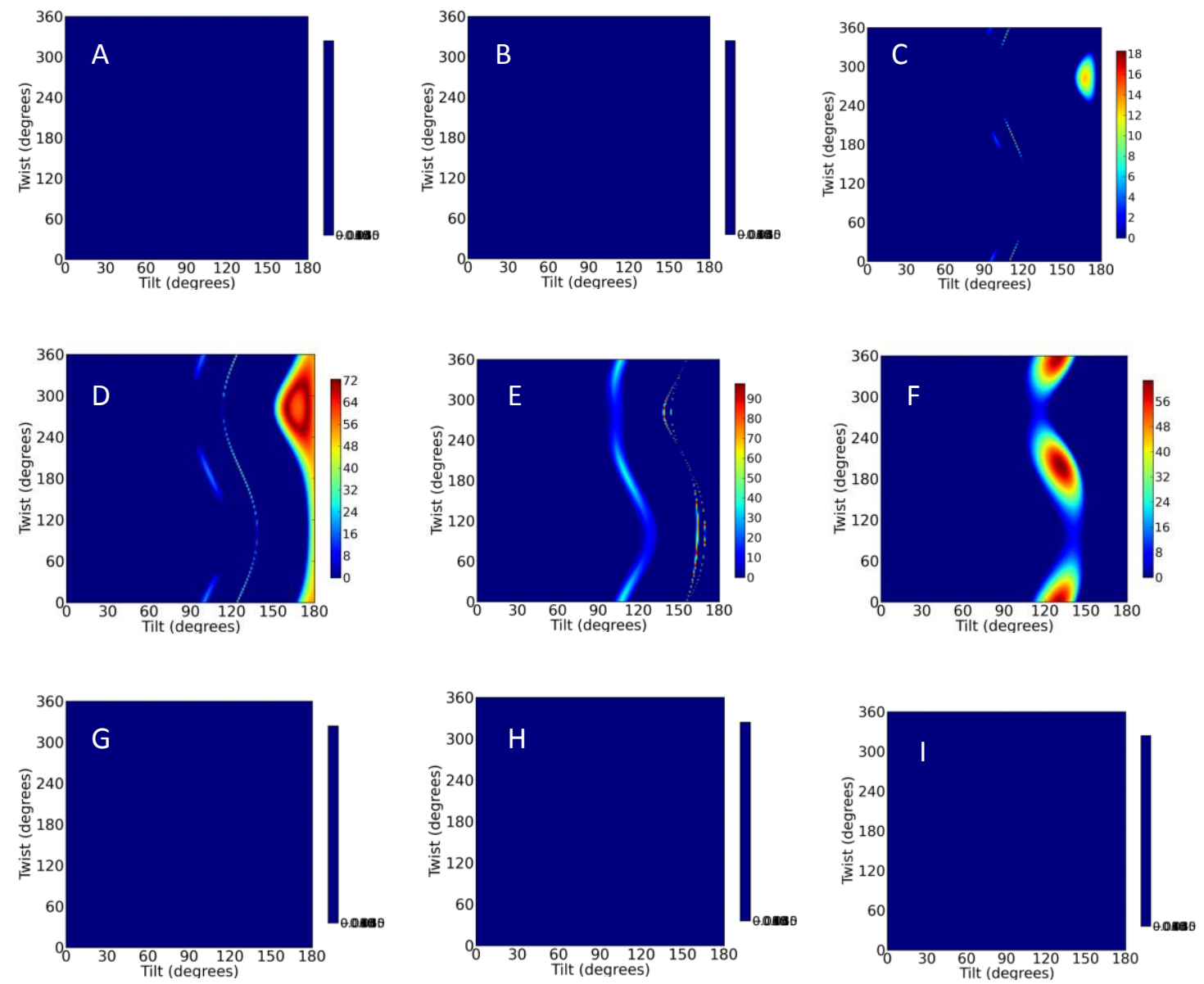

Figure 6 Possibilities of the second orientation of the dual delta distributions for $\mathrm{NfsB}$ (V424C). A: 10\%, B: 20\%, C: 30\%, D: 40\%, E: 50\%, F: 60\%, G: 70\%, H: 80\%, I: 90\% of the NsfB molecule adopt the $(25,30)$ orientation, while the maps show the possibilities of another orientation adopted by the rest of the enzymes. 

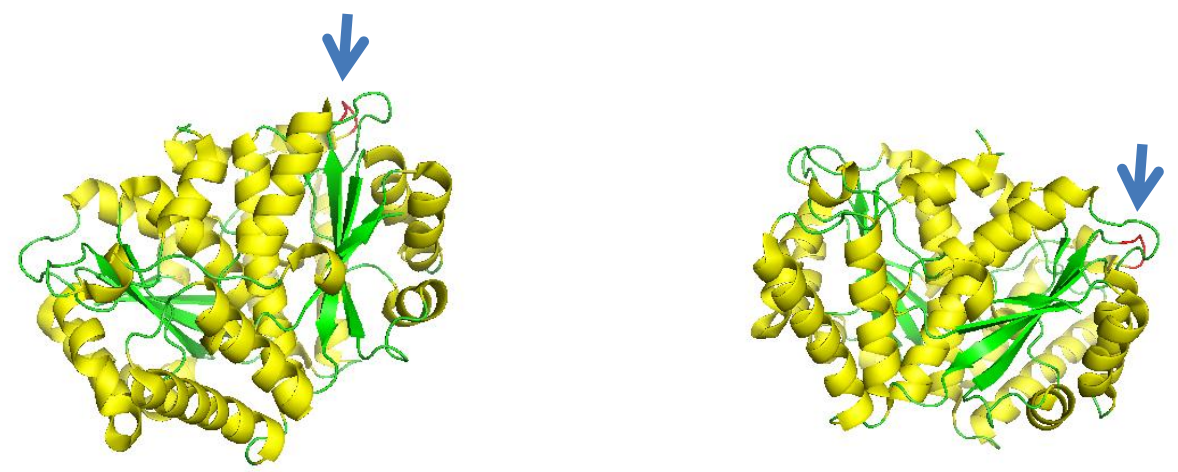

Figure 7 Schematic illustration of a representative second possible orientation of NsfB (V424C) immobilized on the polymer surface. Left: The second orientation $(170,300)$ (60\%) of the immobilized NfsB (V424C) when $40 \%$ of the immobilized enzyme molecules adopt the $(25,30)$ orientation (same as those on SAM). Valine group is labeled in dark, pointing away from the surface. Right: The second orientation $(130,280)(40 \%)$ of the immobilized NfsB (V424C) when $60 \%$ of the immobilized enzyme molecules adopt the $(25,30)$ orientation. Valine 424 is labeled in red too.

H360C

SAM

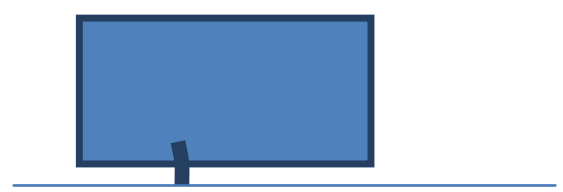

Polymer
V424C

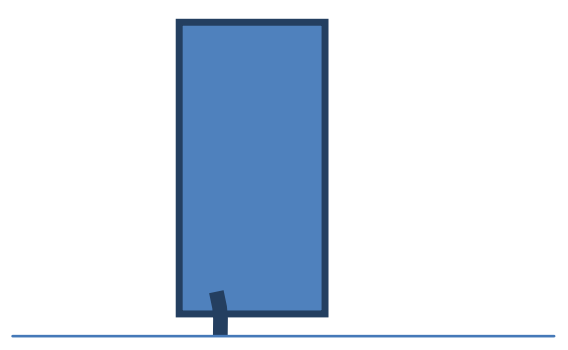

Not Stable

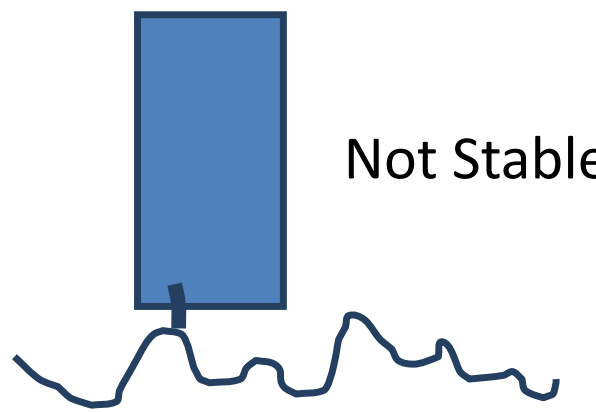

Figure 8 Final orientation of two NsfB constructs on the polymer surface. 


\section{Graphical Abstract}
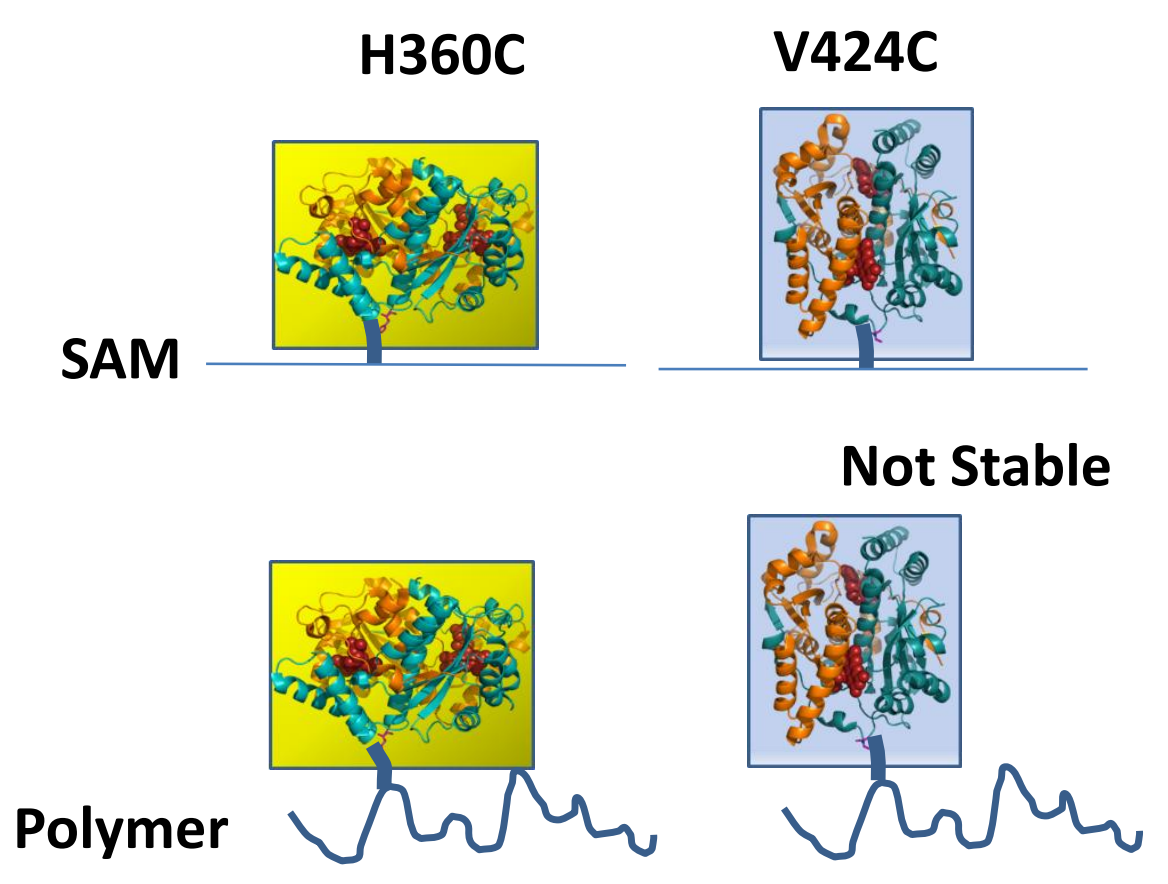